\title{
marges Marges
}

revue d'art contemporain Revue d'art contemporain

03 | 2004

La place de l'image dans le monde contemporain

\section{Éditorial}

Jérôme Glicenstein

\section{(2) OpenEdition}

Journals

Édition électronique

URL : http://journals.openedition.org/marges/754

DOI : 10.4000/marges.754

ISSN : 2416-8742

Éditeur

Presses universitaires de Vincennes

Édition imprimée

Date de publication : 15 novembre 2004

Pagination : 2-4

ISBN : 978-2-84292-247-4

ISSN : $1767-7114$

Référence électronique

Jérôme Glicenstein, « Éditorial », Marges [En ligne], 03 | 2004, mis en ligne le 06 août 2014, consulté le 22 septembre 2020. URL : http://journals.openedition.org/marges/754 ; DOI : https://doi.org/10.4000/ marges.754

Ce document a été généré automatiquement le 22 septembre 2020.

(C) Presses universitaires de Vincennes 


\title{
Éditorial
}

\author{
Jérôme Glicenstein
}

1 Ce troisième numéro de Marges est très largement consacré à la place de l'image dans le monde contemporain. C'est le cas en premier lieu des trois textes et de l'introduction qui nous viennent de la journée d'études proposée par François Soulages à la Maison des Sciences de l'Homme Paris-Nord, en novembre 2003, autour des « Arts et industries des corps et de leurs images ». Ce séminaire de troisième cycle, nous est-il rappelé en introduction, avait pour ambition de mieux comprendre « comment les arts travaillent les corps et leurs images et comment les créations de l'art contemporain se développent à partir des changements fondamentaux actuels relatifs aux corps et à leurs images ». Ainsi, Audrey Leblanc, dans «Un art de la trace du regard », traite des ambivalences de la photographie de reportage et de ce que celle-ci nous dit de "l'autre »; nous présentant notamment le travail de Kyoichi Tzusuki - photographe se considérant comme journaliste, quoique exposant dans des galeries - comme un art exemplaire de la « trace du regard ; un art qui serait en somme « attentif à l'attente de l'autre pour faire sens». Catherine Couanet, quant à elle, dans «Le texte, l'image, l'interprétation ", s'intéresse à la relation entre image et désir à travers trois formes qu'elle qualifie de «toucher-total», de «toucher-divisé » et de « contre-toucher». Ces formes sont mises à profit dans une analyse de la photographie érotique, avec les exemples de l'Homme nu à bicyclette d'Étienne-Jules Marey, les vues stéréoscopiques d'Auguste Belloc et l'Unica de Hans Bellmer. Julia Nyikos enfin revient à un débat beaucoup plus ancien, celui de la relation entre image et écriture à travers l'exemple canonique du Chef d'œuvre inconnu. «Image et invisibilité » traite de la tradition de la description littéraire - l'ekphrasis - telle qu'elle se retrouve dans la nouvelle de Balzac, en y distinguant plusieurs niveaux de compréhension.

2 La pratique de l'ekphrasis n'est d'ailleurs pas très éloignée des préoccupations d'Emanuela Genesio à propos de la relation entre l'écrivaine Anna Banti et la peintre Artemisia Gentileschi. La « rencontre entre récit et peinture » dont il est question est bien entendu "à sens unique " et elle met en jeu d'abord l'identification de la romancière au personnage historique. Mais, comme nous le montre l'auteure, cette relation n'est jamais simple et elle renvoie également à la manière d'écrire l'histoire de l'art : une histoire qui s'apparente bien souvent à une fiction. La conscience de cette 
distance peut alors se révéler féconde; engageant l'écrivaine elle-aussi à «faire œuvre ».

Laetitia Tura n'est pas non plus très éloignée des thématiques abordées lors de la journée d'étude, lorsqu'elle s'interroge dans deux textes (dont l'un écrit en collaboration avec Cédric Deguilhem), à la fois sur ce qu'il est possible de montrer aujourd'hui, et sur les stéréotypes que véhiculent bien souvent « l'imagerie des lieux de conflits ». Son expérience de visite du Liban lui inspire notamment des réflexions sur l'incapacité des images à témoigner du réel ; réflexions qu'elle complète de remarques sur d'autres lieux et d'autres contextes, ainsi que de photos réalisées au Liban. Refusant le « silence éthique » d'Alfredo Jaar, elle nous invite enfin « à reconstruire le récit pour garder mémoire de ce qui s'est passé ».

Dans un registre très différent, Nathalie Fougeras nous parle elle aussi de l'image contemporaine, mais en se situant résolument du côté de l'élaboration fictionnelle propre à la vidéo numérique et aux jeux vidéo. «La caméra et son en_jeu actuel » traite des nouveaux usages permis par le numérique, avec ce que l'auteure désigne comme des relations inédites entre le spectateur et la prise de vue. La "caméra-graphe », la « caméra code » et l'idée d'une "caméra et son univers partagé » dessinent ainsi des nouvelles formes relationnelles, où les images déjouent la relation traditionnelle entre spectateur et œuvre.

5 Le dernier texte - «L'art postmoderne comme idéologie réactionnaire » -, s'éloigne en revanche de cette problématique de l'image, en revenant sur une polémique qui avait fait un certain bruit il y a une vingtaine d'années à propos de la validité de l'art contemporain. Maxence Alcalde replace les termes du débat entre un certain nombre de ses protagonistes passés ou présents, Habermas, Danto, Lyotard, Baudrillard, McEvilley, et remarque qu'il y a eu bien souvent confusion sur l'objet même de la polémique: s'agissait-il d'une discussion touchant à l'art ou à la philosophie ? À l'esthétique ou à l'éthique? Quoi qu'il en soit, ce malentendu a pu perdurer en ressuscitant régulièrement sous d'autres formes jusqu'à ces dernières années.

Enfin, Marges ne renonce pas à publier des comptes rendus d'ouvrages et d'expositions représentatifs de la période qui vient de s'écouler. Dans ce numéro nous avons donc un commentaire à propos de l'ouvrage - qu'il est difficile de qualifier de catalogue publié à l'occasion de l'exposition de Sophie Calle au Centre Pompidou; ouvrage mis en relation avec L'Invité mystère de Grégoire Bouillier. Catherine Couanet a consacré un article aux expositions d'ouverture du Centre national de la photographie au Jeu de Paume. Une autre exposition fait l'objet d'une étude : celle qui inaugurait la Maison rouge, nouvelle fondation d'art contemporain parisienne ouverte en juin dernier. Et une notice est consacrée à La Relation comme forme, un ouvrage de Jean-Louis Boissier qui rend compte de vingt ans d'expérimentations autour de la question de l'interactivité. 